Proceeding Series of the Brazilian Society of Computational and Applied Mathematics

\title{
Estimação de Atitude com Parâmetros Modificados de Rodrigues e dados Reais do Satélite CBERS-2B
}

\section{Roberta Veloso Garcia ${ }^{1}$}

Escola de Engenharia de Lorena, USP, Lorena, SP

Hélio Koiti Kuga ${ }^{2}$

Instituto Nacional de Pesquisas Espaciais, INPE, São José dos Campos, SP

Maria Cecília Zanardi ${ }^{3}$

Centro de Engenharia, Modelagem e Ciências Sociais, UFABC, Santo André, SP

Resumo. A proposta deste trabalho é estudar o comportamento do Filtro de Kalman Unscented (FKU) utilizando os parâmetros modificados de Rodrigues (PMR), quando dados reais de sensores que estão à bordo do satélite CBERS-2B (China Brazil Earth Resources Satellite) alimentam o estimador.

Palavras-chave. Estimação de Atitude, Dados Reais, Filtro de Kalman Unscented, Parâmetros Modificados de Rodrigues

\section{Filtro de Kalman Unscented e Parâmetros Modificados de Rodrigues}

A atitude de um satélite representa como ele está orientado no espaço em relação a um sistema de referencia. Isto está diretamente relacionado com a interpretação de informações obtidas por experimentos realizados com o satélite. No caso particular dos satélites de sensoriamento remoto, como é o caso do satélite CBERS-2B, os dados de atitude são de fundamental importância no processamento de imagens. Para que a missão a que o satélite se destina tenha êxito, o Sistema de Controle de Atitude requer estimativas da atitude em tempo real para que ele possa corrigir o apontamento do satélite conforme desejado. Essas estimativas são produzidas pelo Sistema de Determinação de Atitude, o qual se constitui basicamente de sensores e de métodos de estimação. Dos estimadores de estado, o Filtro de Kalman Unscented (FKU) é um estimador que vem sendo estudado com o intuito de avaliar qual o nível de aplicabilidade em problemas de estimação de atitude. $\mathrm{O}$ FKU é um estimador de estados de sistemas não lineares, e adota a estrutura do Filtro de Kalman (FK) padrão. No entanto, o FKU não se utiliza de linearizações do modelo para

\footnotetext{
${ }^{1}$ robertagarcia@usp.br

${ }^{2}$ helio.kuga@inpe.br

${ }^{3}$ maria.zanardi@ufabc.edu.br
} 
calcular as médias e covariâncias do sistema, mas as calcula por meio da transformação Unscented (TU). A TU consiste em um método de aproximações de média e covariância de variáveis aleatórias transformadas pela própria função não linear do modelo, com o objetivo de contornar os problemas advindos da linearização por truncamento da série de Taylor utilizada nas outras vertentes do FK, [2]. A formulação do filtro é baseada no vetor de estado composto pela atitude, representada pelos parâmetros modificados de Rodrigues (PMR), e pelo bias do giroscópio. A escolha dos PMR se deve a algumas propriedades como, por exemplo, constituir um conjunto mínimo de parâmetros de 3 variáveis que descrevem a orientação de um corpo rígido e são não singulares para qualquer outra rotação múltipla de $2 \pi$. Neste trabalho os PMR representarão o erro da atitude em vez da atitude global, evitando assim as singularidades. Vários artigos estão presentes na literatura contemplando a aplicação do PMR e FKU em problemas de estimação de atitude. No entanto, estes estudos fazem uso de dados simulados de sensores. A contribuição deste trabalho é estudar o comportamento do FKU com PMR, quando dados reais alimentam o filtro. A utilização de dados reais impõem algumas dificuldades como, por exemplo, erros de natureza desconhecida, poucas medidas ou até mesmo medidas corrompidas.

Os PMR são definidos em termos dos quaternions $\mathbf{q}=\left[\begin{array}{ll}\varrho & q_{4}\end{array}\right]^{T}$ como $[1]$

$$
\mathbf{p}=\frac{\varrho}{1+q_{4}}=\mathbf{e} \tan \left(\frac{\varphi}{4}\right)
$$

onde e é o eixo de rotação, $\varphi$ é o ângulo de rotação. O erro da atitude será descrito pelos PMR, dado por [1]

$$
\delta \mathbf{p}=f \frac{\delta \varrho}{a+\delta q_{4}}
$$

$\operatorname{com} \delta \mathbf{q} \equiv\left[\begin{array}{ll}\delta \varrho^{T} & \delta q_{4}\end{array}\right]^{T}$ descrito em [3], a é um parâmetro de 0 à 1 , e $f=2(a+1)$ um fator escalar.

Os resultados mostram que o FKU é eficiente mesmo diante de um número maior de equações (advindas da escolha do PMR) e da utilização de dados reais de sensores de atitude embarcados no satélite.

\section{Agradecimentos}

Os autores agradecem o suporte financeiro recebido pela FAPESP $n^{\circ}(2012 / 21023-6)$.

\section{Referências}

[1] Crassidis J. L., Markley F. L., Unscented filtering for spacecraft attitude estimation, Journal of Guidance, Control and Dynamics, vol.26, 12-28, (2003).

[2] Julier S.J., Uhlmann J.K., Unscented Filtering and Nonlinear Estimation, Proceedings of the IEEE, vol.92, 401-422, (2004).

[3] Lefferts, E. L, Markley, F. L., \& Shuster, M. D., Kalman Filtering for Spacecraft Attitude Estimation, Journal of Guidance, vol.5, 417-429, (1982). 